ARTICLE

Received 5 Dec 2014 | Accepted 29 Apr 2015 | Published 8 Jun 2015

\title{
Emergence of spin-orbit fields in magnetotransport of quasi-two-dimensional iron on gallium arsenide
}

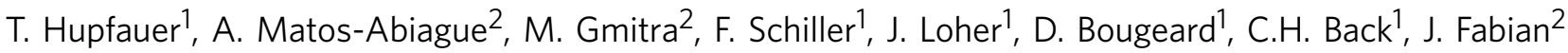 \\ $\&$ D. Weiss ${ }^{1}$
}

The desire for higher information capacities drives the components of electronic devices to ever smaller dimensions so that device properties are determined increasingly more by interfaces than by the bulk structure of the constituent materials. Spintronic devices, especially, benefit from the presence of interfaces-the reduced structural symmetry creates emergent spin-orbit fields that offer novel possibilities to control device functionalities. But where does the bulk end, and the interface begin? Here we trace the interface-to-bulk transition, and follow the emergence of the interfacial spin-orbit fields, in the conducting states of a few monolayers of iron on top of gallium arsenide. We observe the transition from the interface- to bulk-induced lateral crystalline magnetoanisotropy, each having a characteristic symmetry pattern, as the epitaxially grown iron channel increases from four to eight monolayers. Setting the upper limit on the width of the interface-imprinted conducting channel is an important step towards an active control of interfacial spin-orbit fields.

\footnotetext{
${ }^{1}$ Institute of Experimental and Applied Physics, University of Regensburg, 93040 Regensburg, Germany. ${ }^{2}$ Institute of Theoretical Physics, University of Regensburg, 93040 Regensburg, Germany. Correspondence and requests for materials should be addressed to D.W.

(email: dieter.weiss@physik.uni-regensburg.de).
} 
$\mathrm{n}$ recent years, spintronics research ${ }^{1}$ has been fuelled by a new class of experiments related to the combined interaction of the spin and orbital degree of freedom ${ }^{2-10}$. The reduced structural symmetry at surfaces and interfaces gives rise to emergent spin-orbit fields $(\mathrm{SOF})^{11}$. Interfacial SOF are important for phenomena such as tunnelling anisotropic magnetoresistance $(\mathrm{AMR})^{12,13}$, current driven torques at interfaces ${ }^{7,8,14-16}$, formation of two-dimensional magnetic skyrmion lattices ${ }^{17}$ or the realization of topological superconductors ${ }^{18}$.

Owing to the lack of space inversion symmetry at the interface, the electronic structure changes qualitatively-the electronic states are spin split. Unlike a magnetic field, the SOF depend on the electron momentum $\mathbf{k}$, ensuring the time reversal invariance of spin-orbit coupling. Two most common SOFs are (two-dimensional) Dresselhaus ${ }^{19,20}, \mathbf{w}_{\mathrm{D}}=\beta\left(k_{y}, k_{x}\right)$, and the Bychkov-Rashba ${ }^{21,20}, \quad \mathbf{w}_{\mathrm{BR}}=\alpha\left(-k_{y}, \quad k_{x}\right)$ fields, originally introduced to describe the spin-orbit coupling effects in twodimensional electron gases formed in zinc-blende semiconductors grown along [001]. Real parameters $\alpha$ and $\beta$ characterize the strength of the two fields. Combined, the two fields describe a more general $\mathrm{C}_{2 \mathrm{v}}$ SOF appearing in solids with the $\mathrm{C}_{2 \mathrm{v}}$ point symmetry $^{20}$.

Fe on GaAs, grown along [001] has $\mathrm{C}_{2 \mathrm{v}}$ symmetry, induced by the GaAs surface. The same SOFs are also the basis for the evolution of the magnetocrystalline anisotropy in ultra-thin films of $\mathrm{Fe} / \mathrm{GaAs}(001)$ (refs 22,23). Electrons in $\mathrm{Fe}$ should then experience the Dresselhaus and Bychkov-Rashba fields as well. For structures with thick Fe layers (for which magnetotransport was previously studied ${ }^{24,25}$ ), the interface-induced SOF would be hardly noticed, but for ultra-thin films of Fe, consisting of a few atomic monolayers, the interfacial symmetry could even dominate. This is precisely what we observe in our samples of epitaxially grown $\mathrm{Fe}$ on GaAs. As the thickness of $\mathrm{Fe}$ layers decreases, the symmetry of the AMR, which is a signature of spin-orbit coupling, evolves from four-fold-Fe bulk like-to two-fold $\mathrm{C}_{2 \mathrm{v}}$-interface like. We observe reorientation of the main symmetry axes when decreasing the Fe thickness, as well as when increasing temperature. The simple d.c.-transport and measured AMR, presented below, give direct evidence of the presence of the interfacial SOFs. A phenomenological theory, motivated and supported by first-principles calculations, yields perfect fits to the experimental data, allowing us to quantify both the interface and bulk contributions to AMR.

\section{Results}

Experimental set-up. We studied four, six and eight monolayers of Fe epitaxially grown by molecular beam epitaxy (MBE) onto a $100-\mathrm{nm}$ thick layer of undoped GaAs. The mesa structures fabricated from these Fe/GaAs heterostructures, shown in Fig. 1, were defined employing optical lithography and ion beam etching. We used two geometries-a quadrant type sample with the transport channel aligned along seven different crystallographic directions shown in Fig. 1a and an L-shaped one with the transport channels aligned in two perpendicular directions, displayed in Fig. 1d.

For both sample types, the Hall bar width is $40 \mu \mathrm{m}$ and the separation of the potential probes is $50 \mu \mathrm{m}$. For each Fe film thickness, we fabricated and investigated several devices that all show basically the same result.

The AMR measurements were carried out in a variable temperature insert of a ${ }^{4} \mathrm{He}$ cryostat at temperatures ranging from $T=1.8$ to $150 \mathrm{~K}$. The samples were mounted in a sample holder, rotatable by $360^{\circ}$ in an in-plane constant external magnetic field produced by superconducting magnet coils. The angles $\theta$ and $\phi$ in the plane of the film (see Fig. 1b) refer,
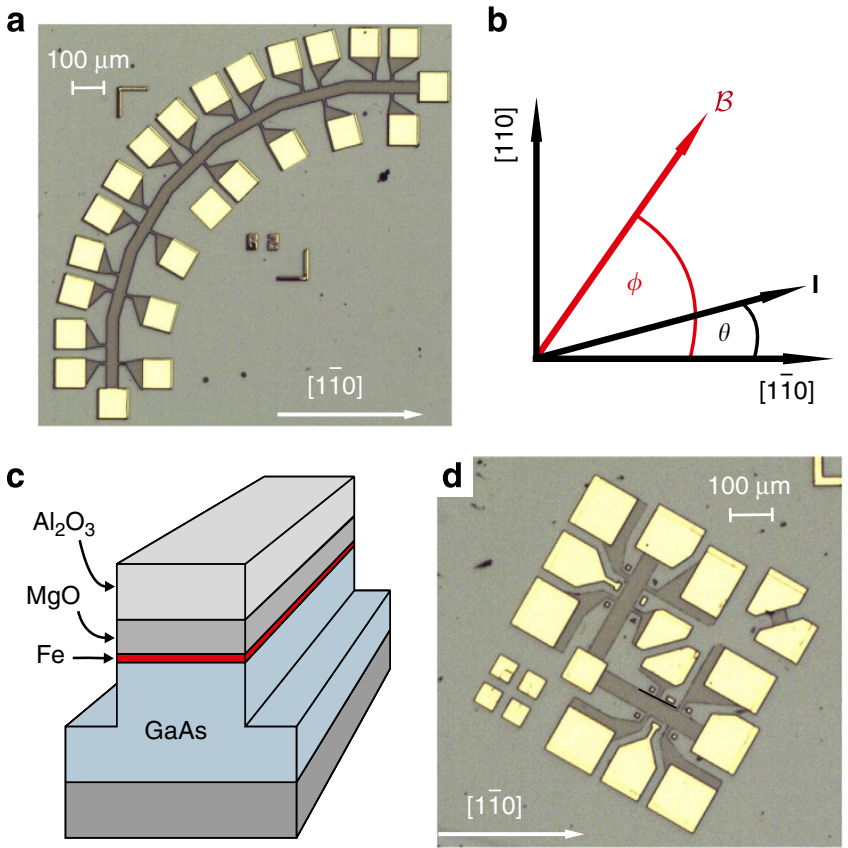

Figure 1 | Design of the Fe/GaAs devices. (a) Optical micrograph of the quadrant type sample after etching and contact metallization. The mesa, defining the transport channel (current direction), is aligned along seven different crystallographic directions allowing to measure simultaneously the corresponding voltage drops. The [110] direction is given by the white arrow and the white bar defines the length scale. (b) The angles $\theta$ and $\phi$ define, respectively, the direction of the current $\mathbf{I}$ and the magnetic field $\mathcal{B}$ with respect to the hard magnetic axis ([110]). (c) Sketch of the mesa and the layer sequence. (d) Optical micrograph of an L-shaped sample after etching and contact metallization. To cover all crystallographic directions in $15^{\circ}$-steps 6 of these structures with different $\theta$ were fabricated.

respectively, to the directions of the current path and the magnetic field $\mathcal{B}$ with respect to the $[1 \overline{1} 0]$-direction of the GaAs crystal. To vary the current direction $\theta$ by $360^{\circ}$, we used, besides the quadrant sample, shown in Fig. 1a, the L-shaped ones, shown in Fig. 1d. By reversing the current direction, we obtain with two quadrant samples a rotation of $\mathbf{j}$ in steps of $15^{\circ}$; using the L-shaped geometry instead, six different samples are needed to cover the full $\theta$ dependence.

First-principles calculations. To gain insight into what anisotropy of the electronic structure can be expected from the Fe/ GaAs interface, we have performed first-principles calculations (see also Supplementary Note 1, Supplementary Figs 1 and 2) of various Fe/GaAs slabs. Figure 2 shows the results for a slab with nine Fe monolayers. The electronic density $\rho(z)$, averaged in the $(x, y)$ plane, is shown in parallel to the atomic structure. The orientation of the axes is as follows: $x=[1 \overline{1} 0], y=[110]$ and $z=[001]$, as in the experiment Fig. 1. To quantify the $\mathrm{C}_{2 \mathrm{v}}$ anisotropy due to the interface, we introduce two measures-the anisotropy of the charge density,

$$
\rho_{\mathrm{A}}(z)=\int|\rho(x, y, z, m)-\rho(y, x, z, m)| \mathrm{d} x \mathrm{~d} y,
$$

and the magnetoanisotropy of the charge density

$$
\rho_{\mathrm{MA}}(z)=\int\left|\rho\left(x, y, z, m_{[110]}\right)-\rho\left(x, y, z, m_{[1 \overline{1} 0]}\right)\right| \mathrm{d} x \mathrm{~d} y .
$$

Here $m$ denotes the orientation of the magnetization. The formula for $\rho_{\mathrm{A}}$ is motivated by the fact that interchanging the 
principal symmetry axes $x$ and $y$ is not a symmetry operation of $\mathrm{C}_{2 \mathrm{v}}$, while it is under the four-fold $\mathrm{C}_{4 \mathrm{v}}$. The magnetoanisotropy $\rho_{\mathrm{MA}}$ is determined by the differences in the charge densities when the orientation of magnetization changes from [110] to [110]. For a four-fold symmetry, both $\rho_{\mathrm{A}}$ and $\rho_{\mathrm{MA}}$ vanish. The anisotropy $\rho_{\mathrm{A}}$ is sensitive mainly to the orbital effects of the interface, while magnetoanisotropy $\rho_{\mathrm{MA}}$ is due to the interfacial spin-orbit coupling. Both $\rho_{\mathrm{A}}(z)$ and $\rho_{\mathrm{MA}}(z)$ are shown in Fig. $2 \mathrm{~b}$, relative to the charge density $\rho(z)$, for the states in a $10-\mathrm{meV}$ window at the Fermi level. The anisotropies are about $10 \%$. The largest anisotropy is at the interfacial $\mathrm{Fe}$ monolayer. In fact, it is the penetration of the Fe Fermi-level Bloch states into the
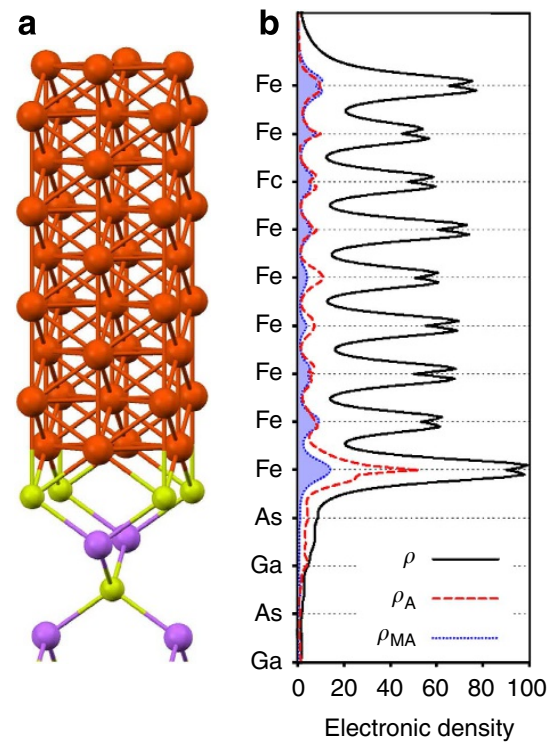
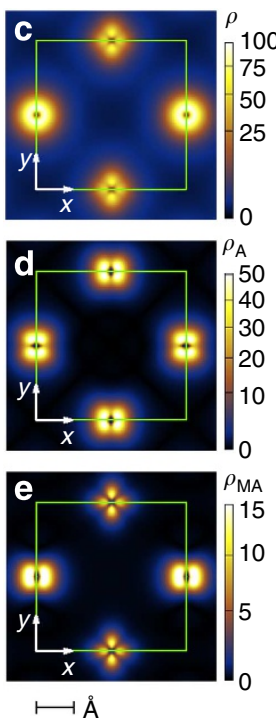

Figure 2 | Anisotropy of the $\mathrm{Fe} / \mathrm{GaAs}$ interface from first principles.

(a) Atomic structure of the interface with nine Fe layers. (b) Calculated electronic density $\rho(z)$, anisotropic electronic density $\rho(z)_{\text {A }}$ and magnetoanisotropic electronic density $\rho(z)_{\mathrm{MA}}$ for the $10-\mathrm{meV}$ window at the Fermi level. The plots are rescaled by the same factor to assign the value of 100 to the maximum of $\rho(z)$. (c), $\rho(x, y)$ (which sets the scale in the same way), (d) $\rho(x, y)_{\mathrm{A}}$ and $(\mathbf{e}) \rho(x, y)_{\mathrm{MA}}$ in the plane of the interfacial Fe atom. The largest densities occur in the vicinity of the four Fe atoms within a unit cell (green). Anisotropic charge density $\rho_{\mathrm{A}}$ is calculated for magnetization along [110]. interface region that imprints the interfacial $\mathrm{C}_{2 \mathrm{v}}$ symmetry onto the lateral Fe transport. This penetration is nicely seen in Fig. $2 b$. As the Fe thickness decreases, the two-fold anisotropies steadily increase (see Supplementary Note 1, Supplementary Table 1). In Fig. $2 \mathrm{c}-\mathrm{e}$, we plot the cross-sectional charge density $\rho(x, y)$, and the corresponding anisotropies $\rho(x, y)_{\mathrm{A}}=\mid \rho(x, y, m)-\rho(y, x$, $m) \mid$ for $m=[110]$ and $\rho(x, y)_{\mathrm{MA}}=\mid \rho(x, y, m=x)-\rho(x, y$, $m=y) \mid$ for the plane of the interfacial Fe, respectively, resolving the $\mathrm{d}$-character of the atomic orbitals of Fe. The existence of SOFs in $\mathrm{Fe} / \mathrm{GaAs}$ was demonstrated from first principles in ref. 26, ramifications for magnetooptics were worked out in ref. 27.

Anisotropic resistance and magnetoresistance. Typical experimental results are displayed in Fig. 3. The resistance averaged over all magnetization directions depends on the crystallographic direction and is always smallest for $\mathbf{j}$ flowing along the $[1 \overline{1} 0]$ direction $\left(\theta=0^{\circ}\right)$. This is shown for devices with six-monolayer Fe in Fig. 3a, for which the data were taken from eight different L-shaped samples. The four-monolayer sample shows a similar resistance anisotropy, while the change in resistance of the eight-monolayer device is comparable to the noise level. This anisotropy of the average resistance is expected and stems, as is pointed out below, from the anisotropy of the charge density $\rho_{\mathrm{A}}$ at the $\mathrm{Fe} / \mathrm{GaAs}$ interface. For the six-monolayer sample, displayed in Fig. 3a, the resistance anisotropy is about $4.3 \%$, to be compared with the calculated anisotropy of about $25 \%$ for an ideal Fe/GaAs interface (see Supplementary Table 1). We now turn to the central experimental observation being the $\theta$ and $\phi$ dependence of the AMR, an effect directly connected to the spin-orbit coupling. Figure $3 \mathrm{~b}$ displays the AMR signal, that is, the longitudinal voltage drop as a function of the in-plane magnetization direction $\phi$ for constant current $I=50 \mu \mathrm{A}$. In experiment, the magnetization direction was rotated by a strong external magnetic field of $10 \mathrm{~T}$ strength. The voltages $U(\phi)$ shown correspond to $\theta=0^{\circ}$ and $90^{\circ}$, that is, for a current flowing along the [110] and [110] direction, respectively, and are highlighted in Fig. 3a. The signal measured for both directions exhibits a $\cos ^{2} \phi$ dependence, typical for AMR. Most importantly the AMR is different for the two current directions: its amplitude $Z$ is $\sim 0.736 \mathrm{mV}$ for $\theta=90^{\circ}$ and $\sim 0.628 \mathrm{mV}$ for $\theta=0^{\circ}$. From that we conclude that the AMR depends on the crystallographic direction of the current flow $(\theta)$ allowing us below to map out the symmetry of the interfacial SOFs.
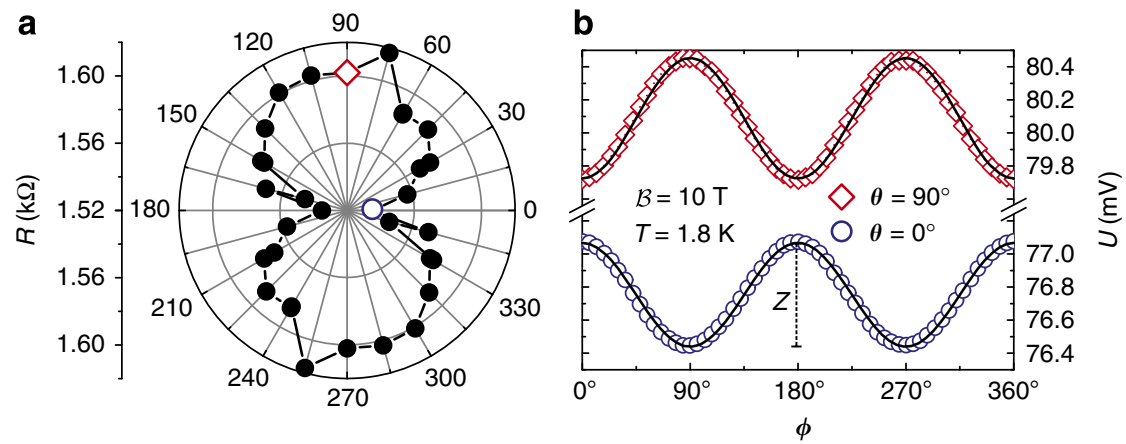

Figure 3 | Resistance and magnetoresistance anisotropy. (a) Average resistance for different current directions $\theta$ of a sample with 6 ML Fe. The resistance anisotropy is $\sim 4.3 \%$. (b) Measurement of the longitudinal voltage drop for a constant current of $50 \mu \mathrm{A}$ as a function of the external magnetic field (magnetization) direction $\phi$, rotated in the interfacial plane. Data were taken at $1.8 \mathrm{~K}$ employing the L-shaped mesas. The two highlighted data points in the polar plot correspond to the two current directions shown in the graph to the right, featuring a difference in the average resistance. Importantly, the AMR effect shows a small but systematic difference in amplitude $Z=U_{\max }-U_{\min }$ for the two current directions along $[1 \overline{1} 0]\left(\theta=0^{\circ}\right)$ and $[110]\left(\theta=90^{\circ}\right)$. The solid lines in $\mathbf{b}$ correspond to a theoretical fit to the experimental data (symbols). 
Crystalline AMR. To quantify the AMR amplitude as a function of $\theta$ we introduce the crystalline AMR (CAMR) coefficient,

$$
\operatorname{CAMR}(\theta)=\frac{U_{\max }(\theta)-U_{\min }(\theta)}{U_{\max }(\theta)+U_{\min }(\theta)},
$$

where $U_{\max }\left(U_{\min }\right)$ refers to the maximum (minimum) value of the voltage drop obtained when $\phi=\theta\left(\phi=\theta+90^{\circ}\right)$. To experimentally extract information about the interfacial SOFs, measurements like the one shown in Fig. $3 b$ were systematically carried out varying the number of Fe monolayers, temperature and direction of current flow $\theta$. We would like to stress that the CAMR effect discussed here originates from the interfacial SOF and not, as, for example, in $(\mathrm{Ga}, \mathrm{Mn}) \mathrm{As}$ layers ${ }^{28-31}$, from bulk spin-orbit interaction.

The measured CAMR as a function of the current direction, $\theta$ is shown in Fig. 4 for different Fe thicknesses and temperatures. The data were taken from two quadrant type samples allowing to change the angle $\theta$ in $15^{\circ}$ steps. In all the cases, the CAMR exhibits a two-fold symmetry with maxima (minima for the case of eight monolayers) for currents flowing along the [1 10$]$ and [110] directions and minima (maxima for the case of eight monolayers) for currents along the [100] and [010] directions. CAMR ranges from 0.2 to $0.4 \%$, decreasing with increasing temperature. The two-fold $\mathrm{C}_{2 \mathrm{v}}$ symmetry component is dominant for the thinnest, four-monolayer, sample. The six-monolayer sample has a strong four-fold component, with the symmetry axes along the $[1 \overline{1} 0]$ and [110] directions, the principal axes of the interfacial $\mathrm{C}_{2 \mathrm{v}}$. Only in the eight-monolayer sample we observe the dominant four-fold symmetry from bulk $\mathrm{Fe}$, with principal axes [100] and [010].

Phenomenological model. The evolution of the symmetry of AMR, from interface to bulk like, with increasing the Fe layer thickness, can be quantitatively described by a phenomenological model which introduces the interfacial $\mathrm{C}_{2 \mathrm{v}}$ SOFs as a perturbation to a bcc (Fe) system. An analogous model was used to describe tunnelling AMR in Fe/GaAs tunnelling devices ${ }^{12,32}$. In short, our system is characterized by two vectors-magnetization $\mathbf{m}$ and SOF $\mathbf{w}(\mathbf{k})$. The momentum-resolved conductivity tensor $g$ can be expanded in the direction cosines of $\mathbf{m}$, as well as in the components of the SOF, up to the second powers. The expansion parameters are restricted by the cubic $\mathrm{O}_{\mathrm{h}}$ symmetry of the unperturbed system; the conductivity tensor also obeys the Onsager relation $g(\mathbf{m})=g^{\mathrm{T}}(-\mathbf{m})$. Averaging over the Fermi momenta, considering that the SOFs are odd in momenta, $\mathbf{w}(\mathbf{k})=-\mathbf{w}(-\mathbf{k})$ (so that averages of terms linear in $\mathbf{w}$ vanish), then gives the conductivity tensor for the lateral transport in $\mathrm{Fe}$, influenced by the SOFs due to the interface. Leaving the details for Supplementary Note 2, we give the result for the longitudinal resistance:

$$
\begin{aligned}
R(\theta, \phi) & \approx A+B \cos ^{2}(\phi-\theta)-\frac{(B-C)}{2} \cos (2 \theta) \cos (2 \phi) \\
& -(D+F+G) \cos (2 \theta)-F \cos (2 \phi) .
\end{aligned}
$$

The constants $A, B$ and $C$, are, in general, different from zero, independent of the presence or not of the interface SOC field. Therefore, they do not account for the interface-induced anisotropy. In particular, $A$ is the isotropic resistance, $B$ is the conventional AMR that depends only on the relative angle between current and magnetization direction; $C$ depends on both the bulk Fe spin-orbit coupling as well as on the magnitude (not direction) of interfacial SOFs, so it does not carry information on the $\mathrm{C}_{2 \mathrm{v}}$ symmetry; $D$ has two contributions, one exclusively determined by the spin-orbit coupling and one combining SOF field and magnetism. Thus, in dependence of the symmetry of the SOF, $D$
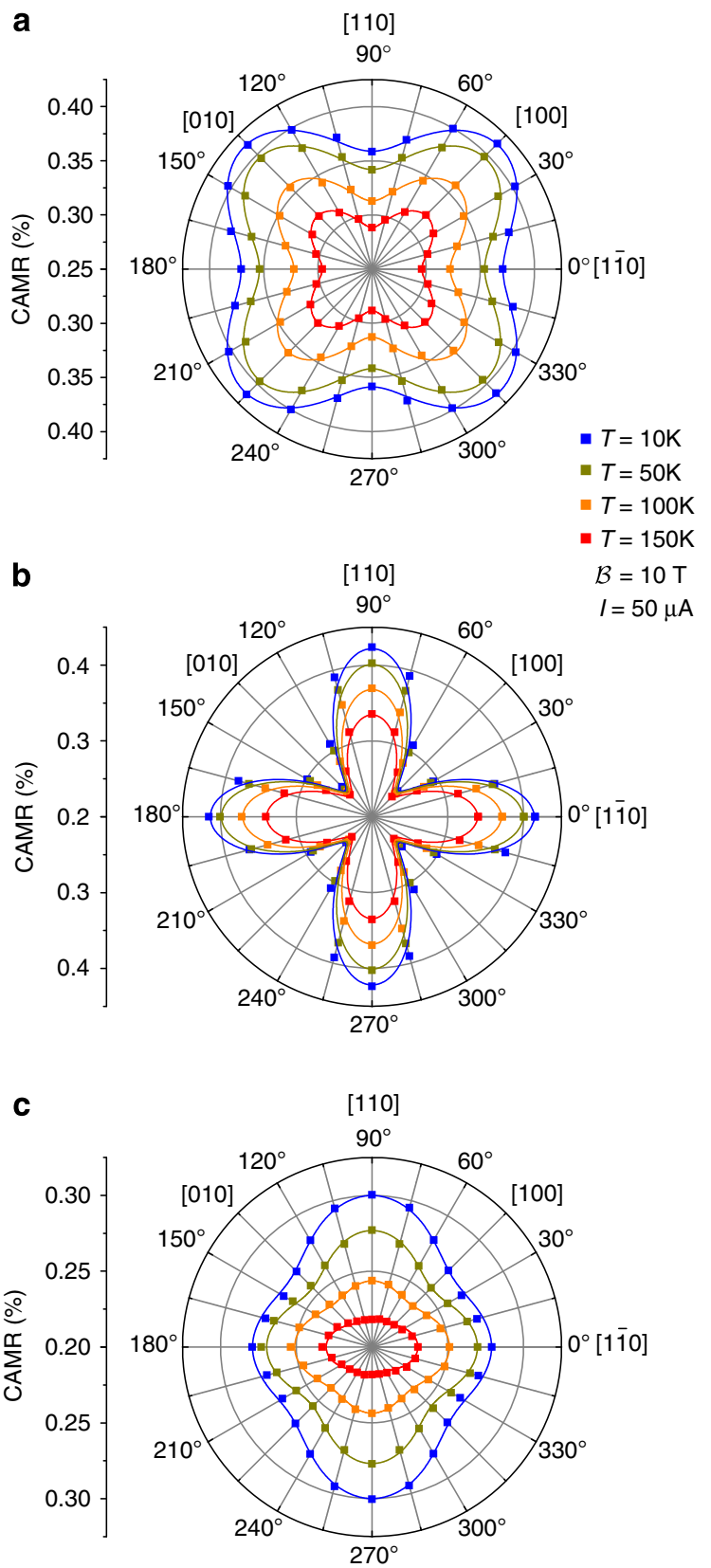

Figure 4 | Crystalline AMR. The CAMR is shown as a function of the direction of the current flow $(\theta)$ at different temperatures. (a-c) correspond to samples with eight, six and four monolayers of Fe, respectively. The symbols are experimental data. The solid lines are fits using our phenomenological model. The difference between the maxima of the CAMR along [110] and [110] accounts for the presence of the interface SOF, which lowers the symmetry from four-fold to two-fold.

can be finite even in the absence of magnetism (it can therefore account for anisotropic transport in two-dimensional electron gases in zinc-blende semiconductors $\left.{ }^{33,34}\right)$. The parameter $F$ contains information of magnetism and SOF. It combines the crystalline anisotropy produced by SOF with the magnetic properties of the system and, as shown below, characterizes the interface-induced CAMR. Finally, $G$ has a purely orbital nature and describes the intrinsic anisotropy of the interfacial structure.

Using the symmetry properties of the Dresselhaus and Bychkov-Rashba SOFs, one finds $D$ and $F$ proportional to the 
product $\alpha \beta$ (refs 20,35). Therefore, for a $\mathrm{C}_{2 \mathrm{v}}$ symmetric SOF (that is, $\alpha \beta \neq 0$ ), the parameters $D$ and $F$ are, in general, finite. In contrast, if the symmetry of the SOC is $\mathrm{C}_{\infty \mathrm{v}}(\beta=0), \mathrm{D}_{2 \mathrm{~d}}(\alpha=0)$ or $\mathrm{C}_{4 \mathrm{v}}$ (terms of higher order in momentum reduce the symmetry of the Bychkov-Rashba SOF to $\mathrm{C}_{4 \mathrm{v}}$ ), both $D$ and $F$ vanish.

\section{Discussion}

The experimentally found behaviour of the CAMR can be understood in terms of our phenomenological model. Using equations (3) and (4), and taking into account that the anisotropic corrections are small, we obtain

$$
\operatorname{CAMR}(\theta) \approx \frac{B+C+(C-B) \cos (4 \theta)-4 F \cos (2 \theta)}{4 A} .
$$

This expression fits perfectly the experimental data in Fig. 4 . Equation (5) provides insight about the microscopic origin of the CAMR. The amplitude of the four-fold symmetric contribution $[\propto \cos (4 \theta)]$ is determined by the phenomenological parameters $B$ and $C$ which, as discussed above, are finite even in the absence of interfacial SOFs. The four-fold term then accounts for the cubic symmetry of the underlying $\mathrm{Fe}$ system in the absence of the interface and is responsible for the appearance of the four lobes in the polar plots of the CAMR (see Fig. 4). On the other hand, the amplitude of the two-fold symmetric contribution $[\propto \cos (2 \theta)]$ is given by the parameter $F$ only which, as discussed above, accounts for the $\mathrm{C}_{2 \mathrm{v}}$ SOFs of the $\mathrm{Fe} / \mathrm{GaAs}$ interface and increases with the wave function penetration into the interface region. This contribution is responsible for the observed difference between the maxima of the CAMR along [11 0$]$ and [110]. In fact, it follows from equations (5) that $\operatorname{CAMR}\left(90^{\circ}\right)-\operatorname{CAMR}\left(0^{\circ}\right) \propto F$.

Our model also explains the reorientation of the four-fold symmetry axes when increasing the Fe thickness from six to eight monolayers (see Fig. 4). Which axes become principal depends on the sign of $C-B$ in equation (5). Both $B$ and $C$ depend on the bulk Fe properties, as well as on the amplitude (but not direction) of the interfacial SOFs. The term corresponding to the bulk Fe properties dominates in thicker samples (eight monolayers), while the interfacial one in thinner samples (six monolayers). The two contributions compete, giving the sign change of $C-B$ when changing the layer thickness, causing reorientation of the CAMR symmetry axes, in agreement with the experimental observation. Further details can be found in the Supplementary Note 3, and the extracted values of the phenomenological parameters and their temperature dependence, are presented in Supplementary Figs 3-5.

In conclusion, we have given experimental evidence, from a simple d.c. transport measurement, of interfacial SOFs in ultrathin Fe on GaAs. We have also given a general phenomenological model that nicely fits all the experimental data, and qualitatively accounts for the observed evolution of the CAMR from interface dominated two-fold, via an intermediate one with the symmetry axes still given by the interfacial symmetry, to the four-fold bulk dominated symmetry, as the thickness of the Fe layer increases from four, through six, to eight monolayers. Our findings open the field of lateral anisotropic magnetotransport in ferromagnetic nanochannels on zinc-blende semiconductors, which has potential technological applications in memory-sensing and memorystorage devices.

\footnotetext{
Methods

Experimental methods. The thin Fe films of varying thickness were grown by MBE at a temperature of $\sim 75^{\circ} \mathrm{C}$ side by side on the same surface by controlling the monolayer growth using a shiftable shutter. The GaAs layer had been grown before in a separate chamber onto a (001)-oriented semi-insulating GaAs substrate using an excess of As, which ensures an As-terminated surface, showing a $(2 \times 4)$ surface reconstruction. The As termination ensures that $\mathrm{Ga}$ diffusion into the $\mathrm{Fe}$ film is kept to a minimum and justifies the assumed As termination in the ab initio
}

calculations. For Fe deposition, the GaAs wafer was transferred into the attached metal MBE chamber. Flat and epitaxial growth of Fe was confirmed by monitoring reflection high-energy electron diffraction oscillations during Fe film growth. To protect the Fe layer from oxidation, it was covered with an $\sim 10$-nm-thick layer of thermally evaporated $\mathrm{MgO}$ and $15 \mathrm{~nm} \mathrm{Al}_{2} \mathrm{O}_{3}$, grown by atomic layer deposition. The mesa structures fabricated from these Fe/GaAs heterostructures were defined employing optical lithography and ion beam etching. The contact pads, also defined by optical lithography and consisting of $15 \mathrm{~nm} \mathrm{Ti}$ and $130 \mathrm{~nm} \mathrm{Au}$, were evaporated on top of the $\mathrm{Al}_{2} \mathrm{O}_{3}$ and contact to the Fe layer was made by ultrasonic bonding with sufficiently high power to penetrate the insulating layers. This results in ohmic contacts with negligible contact resistance of $<1 \mathrm{k} \Omega$, confirmed by comparing two- and four-wire $I-V$ characteristics at cryogenic and room temperature. All these steps, except the final $\mathrm{Al}_{2} \mathrm{O}_{3}$-deposition, were performed without breaking the vacuum.

Theoretical methods. The first-principles calculations reported in the manuscript were performed using the full potential linearized augmented planewave method as implemented in Wien $2 \mathrm{k}$ code $\mathrm{e}^{36}$. The method is an all-electron method within density functional theory ${ }^{37}$. We used a generalized gradient approximation ${ }^{38}$ for the exchange-correlation functional. We considered muffin-tin radii $2.3,1.89$ and 2.3 Bohr radii for $\mathrm{Ga}$, As and $\mathrm{Fe}$, respectively. We used mixed linearized augmented planewave and augmented plane wave + local orbital basis set with plane wave energy cutoff of about 24 Ry. For irreducible part of the first Brillouin zone sampling, we considered $364 k$-points. It turned out that the used parameters yield sufficient accuracy in determining the in-plane magnetocrystalline anisotropy $E([1 \overline{1} 0])-E([110])$ of $0.0065,0.0057$ and $0.0033 \mathrm{mRy}$ for three, six and nine atomic layers of epitaxial Fe on (001) GaAs, respectively. Spin-orbit coupling of the valence electrons has been treated scalar relativistically within the second variational method.

The small lattice mismatch between twice the lattice constant of Fe $(2.87 \AA)$ and GaAs (5.65 $\AA$ ) allows for a smooth epitaxial growth of Fe on a GaAs (001) surface. We took for the diagonal lattice spacing $a / \sqrt{2}=3.997 \mathrm{~A}$. To study the electronic structure of the GaAs/Fe interface, we consider thin ideal Fe/GaAs slabs separated by a vacuum of $13 \AA$ in growth direction (001) to exclude interaction between periodic images. The semiconducting substrate contains 13 atomic layers of GaAs (three unit cells of bulk GaAs) with As-terminated surface and interface. Dangling bonds on surface As atoms have been passivated by two fictitious hydrogen atoms with fractionally reduced proton charge and corresponding fractional electronic charge to $0.75 e$, where $e$ is the elementary charge. In this way, irrelevant surface states are removed from the bandgap region due to the formation of the covalent bonds ${ }^{39,40}$. The As-H bond length of $1.55 \AA$ and angle $112.9^{\circ}$ between $\mathrm{Ga}$, As and $\mathrm{H}$ have been obtained from the surface relaxation. For the epitaxially grown $\mathrm{Fe}$ on (001) GaAs surface, we consider flat $1 \times 1$ interface.

\section{References}

1. Žutić, I., Fabian, J. \& Das Sarma, S. Spintronics: fundamentals and applications. Rev. Mod. Phys. 76, 323-410 (2004).

2. Chernyshov, A. et al. Evidence for reversible control of magnetization in a ferromagnetic material by means of spin-orbit magnetic field. Nat. Phys. 5, 656-659 (2009).

3. Maruyama, T. et al. Large voltage-induced magnetic anisotropy change in a few atomic layers of iron. Nat. Nanotechnol. 4, 158-161 (2009).

4. Fang, D. et al. Spin-orbit-driven ferromagnetic resonance. Nat. Nanotechnol. 6, 413-417 (2011).

5. Fan, X. et al. Observation of the nonlocal spin-orbital effective field. Nat. Commun. 4, 1799 (2013).

6. Fan, X. et al. Quantifying interface and bulk contributions to spin-orbit torque in magnetic bilayers. Nat. Commun. 5, 3042 (2014).

7. Garello, K. et al. Symmetry and magnitude of spin-orbit torques in ferromagnetic heterostructures. Nat. Nanotechnol. 8, 587-593 (2013).

8. Miron, I. M. et al. Current-driven spin torque induced by the Rashba effect in a ferromagnetic metal layer. Nat. Mater. 9, 230-234 (2010).

9. Liu, L., Lee, O. J., Gudmundsen, T. J., Ralph, D. C. \& Buhrman, R. A. Current-induced switching of perpendicularly magnetized magnetic layers using spin torque from the Hall effect. Phys. Rev. Lett. 109, 096602 (2012).

10. Liu, L. et al. Spin-torque switching with the giant spin Hall effect of tantalum. Science 336, 555-558 (2012).

11. Rashba, E. I. Properties of semiconductors with an extremum loop. 1. cyclotron and combinational resonance in a magnetic field perpendicular to the plane of the loop. Fiz. Tverd. Tela (Leningrad) 2, 1224-1238 (1960).

12. Moser, J. et al. Tunneling anisotropic magnetoresistance and spin-orbit coupling in Fe/GaAs/Au tunnel junctions. Phys. Rev. Lett. 99, 056601 (2007).

13. Uemura, T., Imai, Y., Harada, M., Matsuda, K. \& Yamamoto, M. Tunneling anisotropic magnetoresistance in epitaxial $\mathrm{CoFe} / \mathrm{n}-\mathrm{GaAs}$ junctions. Appl. Phys. Lett. 94, 182502 (2009).

14. Miron, I. M. et al. Perpendicular switching of a single ferromagnetic layer induced by in-plane current injection. Nature 476, 189-193 (2011) 
15. Ryu, K.-S., Thomas, L., Yang, S.-H. \& Parkin, S. P. P. Chiral spin torque at magnetic domain walls. Nat. Nanotechnol. 8, 527-533 (2013).

16. Emori, S., Bauer, U., Ahn, S.-M., Martinez, E. \& Beach, G. S. D. Current-driven dynamics of chiral ferromagnetic domain walls. Nat. Mater. 12, 611-616 (2013).

17. Heinze, S. et al. Spontaneous atomic-scale magnetic skyrmion lattice in two dimensions. Nat. Phys. 7, 713-718 (2011).

18. Nadj-Perge, S. et al. Observation of Majorana fermions in ferromagnetic atomic chains on a superconductor. Science 346, 602-607 (2014).

19. Dresselhaus, G. Spin-orbit coupling effects in zinc blende structures. Phys. Rev 100, 580-586 (1955).

20. Fabian, J., Matos-Abiague, A., Ertler, C., Stano, P. \& Žutić, I. Semiconductor spintronics. Acta Phys. Slov. 57, 565-907 (2007).

21. Bychkov, Y. A. \& Rashba, E. I. Properties of a 2 D electron gas with lifted spectral degeneracy. JETP Lett. 39, 78-81 (1984).

22. Wastlbauer, G. \& Bland, J. A. C. Structural and magnetic properties of ultrathin epitaxial Fe films on $\mathrm{GaAs}(001)$ and related semiconductor substrates. Adv. Phys. 54, 137-219 (2005).

23. Urban, R., Woltersdorf, G. \& Heinrich, B. Gilbert damping in single and multilayer ultrathin films: role of interfaces in nonlocal spin dynamics. Phys. Rev. Lett. 87, 217204 (2001).

24. Tondra, M. et al. Thickness dependence of the anisotropic magnetoresistance in epitaxial iron films. J. Appl. Phys. 73, 6393-6395 (1993).

25. Riggs, K. T., Dahlberg, E. D. \& Prinz, G. A. First-order magnetic-field-induced phase transition in epitaxial iron films studied by magnetoresistance. Phys. Rev. B 41, 7088-7095 (1990).

26. Gmitra, M., Matos-Abiague, A., Draxl, C. \& Fabian, J. Magnetic control of spin-orbit fields: a first-principles study of Fe/GaAs Junctions. Phys. Rev. Lett. 111, 036603 (2013).

27. Putz, S., Gmitra, M. \& Fabian, J. Anisotropic optical properties of Fe/GaAs(001) nanolayers from first principles. Phys. Rev. B 90, 045315 (2014).

28. Limmer, W. et al. Angle-dependent magnetotransport in cubic and tetragonal ferromagnets: application to (001)- and (113)A-oriented (Ga,Mn)As. Phys. Rev. B 77, 205205 (2006)

29. Limmer, W. et al. Advanced resistivity model for arbitrary magnetization orientation applied to a series of compressive- to tensile-strained (Ga,Mn)As layers. Phys. Rev. B 77, 205210 (2008).

30. Rushforth, A. W. et al. Anisotropic magnetoresistance components in (Ga,Mn)As. Phys. Rev. Lett. 99, 146207 (2007).

31. De Ranieri, A. et al. Lithographically and electrically controlled strain effects on anisotropic magnetoresistance in (Ga,Mn)As. New J. Phys. 10, 065003 (2008).

32. Uemura, T., Harada, M., Matsuda, K. \& Yamamoto, M. Internal electric field influence on tunneling anisotropic magnetoresistance in epitaxial ferromagnet/n-GaAs junctions. Appl. Phys. Lett. 96, 252106 (2010).

33. Trushin, M. \& Schliemann, J. Anisotropic current-induced spin accumulation in the two-dimensional electron gas with spin-orbit coupling. Phys. Rev. B 75, 155323 (2007)
34. Chalaev, O. \& Loss, D. Spin-orbit-induced anisotropic conductivity of a disordered two-dimensional electron gas. Phys. Rev. B 80, 035305 (2009).

35. Matos-Abiague, A. \& Fabian, J. Anisotropic tunneling magnetoresistance and tunneling anisotropic magnetoresistance: spin-orbit coupling in magnetic tunnel junctions. Phys. Rev. B 79, 155303 (2009).

36. Blaha, P., Schwarz, K., Madsen, G. K. H., Kvasnicka, D. \& Luitz, J. Wien2k, an augmented plane wave + local orbitals program for calculating crystal properties (Vienna University of Technology, 2001).

37. Hohenberg, P. \& Kohn, W. Inhomogeneous electron gas. Phys. Rev. 136, B864-B871 (1964).

38. Perdew, J. P., Burke, K. \& Ernzerhof, M. Generalized gradient approximation made simple. Phys. Rev. Lett. 77, 3865-3868 (1996).

39. Shiraishi, K. A new slab model approach for electronic structure calculation of polar semiconductor surface. J. Phys. Soc. Jpn 59, 3455-3458 (1990).

40. Huang, X., Lindgren, E. \& Chelikowsky, J. R. Surface passivation method for semiconductor nanostructures. Phys. Rev. B 71, 165328 (2005).

\section{Acknowledgements}

We thank Georg Woltersdorf for discussions and acknowledge support from the German Science Foundation (DFG) via SFB 689.

\section{Author contributions}

D.W., J.F., A.M.-A. and T.H. devised the experiment, F.S., J.L., D.B. and C.H.B. were involved in MBE growth; T.H. prepared the samples and performed the measurements; J.F., A.M.-A. and M.G. developed the theory; T.H., A.M.-A., M.G., C.H.B., J.F. and D.W. wrote the paper.

\section{Additional Information}

Supplementary Information accompanies this paper at http://www.nature.com/ naturecommunications

Competing financial interests: The authors declare no competing financial interests.

Reprints and permission information is available online at http://npg.nature.com/ reprintsandpermissions/

How to cite this article: Hupfauer, T. et al. Emergence of spin-orbit fields in magnetotransport of quasi-two-dimensional iron on gallium arsenide. Nat. Commun. 6:7374 doi: $10.1038 /$ ncomms8374 (2015).

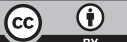

This work is licensed under a Creative Commons Attribution 4.0 International License. The images or other third party material in this article are included in the article's Creative Commons license, unless indicated otherwise in the credit line; if the material is not included under the Creative Commons license, users will need to obtain permission from the license holder to reproduce the material To view a copy of this license, visit http://creativecommons.org/licenses/by/4.0/ 